\title{
Stereospecific Reaction of Donor-Acceptor Cyclopropanes with Thioketones: A Novel Access to Highly Substituted Tetrahydrothiophenes
}

\author{
André U. Augustin, Maximilian Sensse, Peter G. Jones, and Daniel B. Werz* \\ Dedicated to Professor Dr. Stefan Schulz on the occasion of his $60^{\text {th }}$ birthday
}

\begin{abstract}
Lewis-acid-catalyzed reactions of 2-substituted cyclopropane 1,1-dicarboxylates with thioketones are described. Highly substituted tetrahydrothiophenes with two adjacent quaternary carbon atoms were obtained in a stereospecific manner under mild conditions and in high yield using $\mathrm{AlCl}_{3}$ as Lewis acid. Moreover, an intramolecular approach was successfully implemented to gain access to sulfur-bridged [n.2.1] bicyclic ring systems. Conversion of selenoketones, the heavier analogs, under similar conditions resulted in the formation of various tetrahydroselenophenes.
\end{abstract}

The carbonyl group is probably one of the most abundant and versatile functional groups in organic compounds. In contrast, its heavier analogs, thio- and selenoketones, are much less prominent. The carbon-sulfur $\pi$-bond shows a high reactivity because of the poor orbital overlap between two elements that differ significantly in size. On the one hand, this fact makes thiones interesting reagents for organic chemists. On the other hand, the handling of these compounds is challenging since they tend to oligomerize and undergo various side reactions. Presumably, the latter is one reason why their chemical value has been underestimated in the past. Nevertheless, thiocarbonyls have been employed for the construction of sulfur-containing heterocycles. ${ }^{[1]}$ Direct access to substituted thiiranes was realized by a formal $[2+1]$-cycloaddition with carbenes, ${ }^{[2]}$ whereas fourmembered thietanes were accessed from the corresponding olefins, ${ }^{[3]}$ allenes,${ }^{[4]}$ ketenes $^{[5]}$ or arynes ${ }^{[6]}$ in a $[2+2]$-process (Scheme 1). The hetero-Diels-Alder-reaction of thiocarbonyls with dienes is well explored and various $2 \mathrm{H}$-thiopyrans have been obtained. ${ }^{[7]}$ Remarkably, a five-membered ring-formation of thioketones and a three-carbon unit has rarely been reported. ${ }^{[8]}$ Donor-acceptor (D-A) cyclopropanes are one of the most frequently used three-atom building blocks. ${ }^{[9]}$ Their special reactivity is explained by the high ring strain of about $115 \mathrm{~kJ} / \mathrm{mol}$ and the vicinal arrangement of electron-donating and electron-

["] M. Sc. A. U. Augustin, B. Sc. M. Sensse, Prof. Dr. D. B. Werz Technische Universität Braunschweig Institute of Organic Chemistry Hagenring 30, 38106 Braunschweig (Germany) E-mail: d.werz@tu-braunschweig.de

Homepage: http://www.werzlab.de

Prof. Dr. P. G. Jones

Technische Universität Braunschweig

Institute of Inorganic and Analytical Chemistry

Hagenring 30, 38106 Braunschweig (Germany)

Supporting information and the ORCID identification number(s) for the author(s) of this article can be found: accepting substituents. ${ }^{[10]}$ This substitution pattern leads to an easily cleavable bond. The emerging 1,3-zwitterionic intermediates are able to undergo various transformations such as rearrangements, ${ }^{[11]}$ ring-opening reactions with electrophilic and nucleophilic components ${ }^{[12]}$ or cycloaddition reactions. ${ }^{[13]}$ One-, two-, three- and four-atom components were successfully inserted, leading to four-, five-, six- and seven-membered carboand heterocyclic compounds via $(3+n)$ annulation reactions. ${ }^{[11-13]}$ To afford substituted tetrahydrofurans, Johnson and Waser made use of the dipolar $\mathrm{CO}$ double bond in aldehydes and ketones, ${ }^{[14,15]}$ whereas cyclopentane or cyclopentene derivatives were obtained by inserting highly polarized CC double or triple bond systems. ${ }^{[16,17]}$ Whereas these types of annulations are well established and even catalytic, enantioselective protocols have been developed, higher homologs of ketones such as thio- and selenoketones have never been subjected to a [3+2]cycloaddition with D-A cyclopropanes.

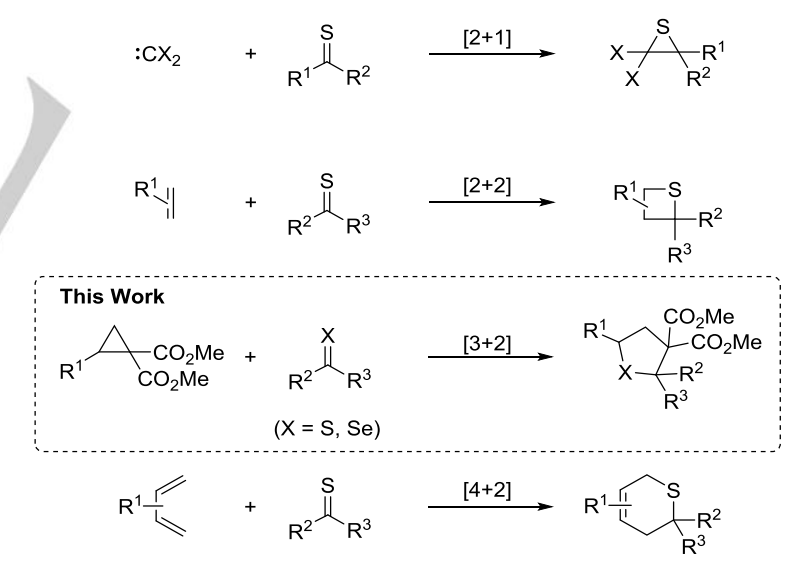

Scheme 1. $[n+2]-C y c l o a d d i t i o n$ processes of thioketones with $C_{n}$ units and our present work using D-A cyclopropanes as three-membered building blocks.

At the outset of our studies, we chose cyclopropane diester 1a and thioketone $\mathbf{2 a}$ as model substrates to optimize the conditions for the anticipated [3+2]-cycloaddition process. As a starting point, the reaction was carried out in dichloromethane at $25^{\circ} \mathrm{C}$ for 8 hours. Whereas most of the commonly used Lewis acids gave only traces or at best small amounts of the desired product (Table 1, entries 1-3), $\mathrm{AlCl}_{3}$ instantly delivered $3 \mathbf{a}$ in moderate yield (entry 4). Further optimization demonstrated that an increase of the amount of thiobenzophenone $\mathbf{2} \mathbf{a}$ has a very beneficial effect and resulted in quantitative product formation (entries 5-6). 
Table 1. Optimization of the reaction conditions. ${ }^{[a]}$

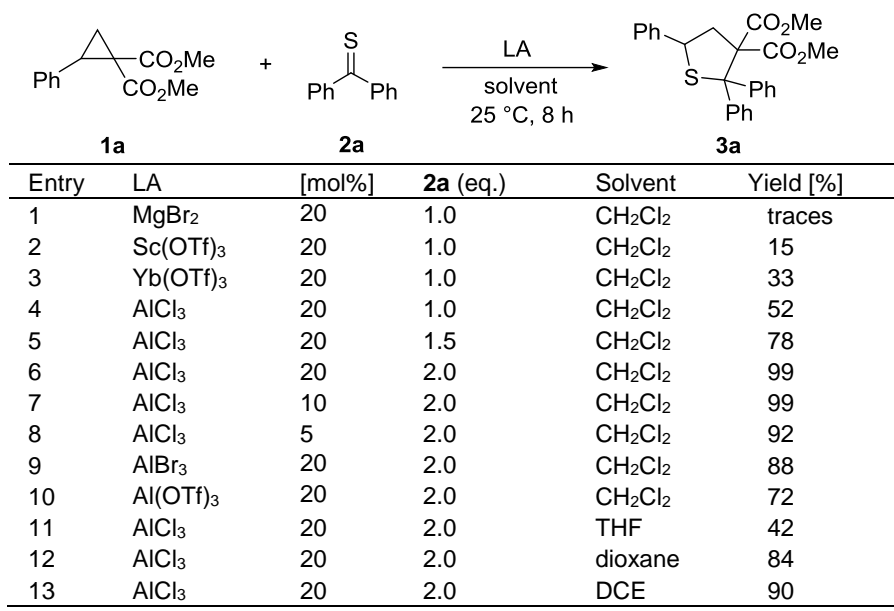

[a] Reaction conditions: 1a $(100 \mu \mathrm{mol}), \mathbf{2 a}$, solvent $(2.0 \mathrm{~mL}), 25^{\circ} \mathrm{C}, 8 \mathrm{~h}, \mathrm{Ar}$ atmosphere; yields represent isolated and purified products; $\mathrm{DCE}=1,2$ dichloroethane.

Next, we decreased the catalyst loading to $10 \mathrm{~mol} \%$ without loss of product formation, even 5 mol\% gave $3 a$ in $92 \%$ yield (entries 7-8). However, the yields dropped significantly when using $\mathrm{AlBr}_{3}$ or $\mathrm{Al}(\mathrm{OTf})_{3}$ as Lewis acid (entries 9-10). Other solvents such as ethers or DCE did not match the yields obtained in dichloromethane (entries 11-13).
With the optimized conditions in hand, we examined the scope of the reaction, first varying the donor $\left(R^{1}\right)$ at the $D-A$ cyclopropane 1 (Scheme 2). Various aryl units bearing different electron-donating and electron-withdrawing substituents (3b-3g and $\mathbf{3 k}$ ) furnished the desired products in up to $99 \%$ yield. Methyl substituents in ortho-, meta- and para-position were also tolerated, as were bulky groups such as naphthyl residues (3h-3j and $\mathbf{3 l}$ ).

Besides aryl groups, weakly electron-donating aliphatic moieties (e.g. cyclohexyl and vinyl residues) were successfully converted into the corresponding THT scaffolds in good yields $(3 m-3 n)$. The transformation also proceeded smoothly with furyl and thienyl donors $(30-3 p)$. Decoration of the three-membered ring with succinimide or phthalimide afforded the desired products $3 q-3 r$ in moderate to good yields.

After these promising results, the generality of the protocol was evaluated using different thiocarbonyls. To our satisfaction, other aliphatic and aromatic thioketones and mixed derivatives performed well under similar conditions. As depicted in Scheme 3, the cycloaddition process worked well for the reaction with bulky adamantanethione and cyclopropenethione to construct spiro compounds $\mathbf{4 a}$ and $\mathbf{4 b}$ in up to $89 \%$ yield. Subsequently, a series of non-symmetric thioketones was subjected to the reaction conditions to afford both the cis- and trans-products in good yields (4da-4ga). Initial attempts under the standard conditions gave both diastereomers in a ratio of about 1:1. Lowering the reaction temperature to $5{ }^{\circ} \mathrm{C}$ furnished the isomers in acceptable diastereoselectivity of up to $4: 1$. NMR experiments by NOE confirmed our expectations that the cis-product is favored.

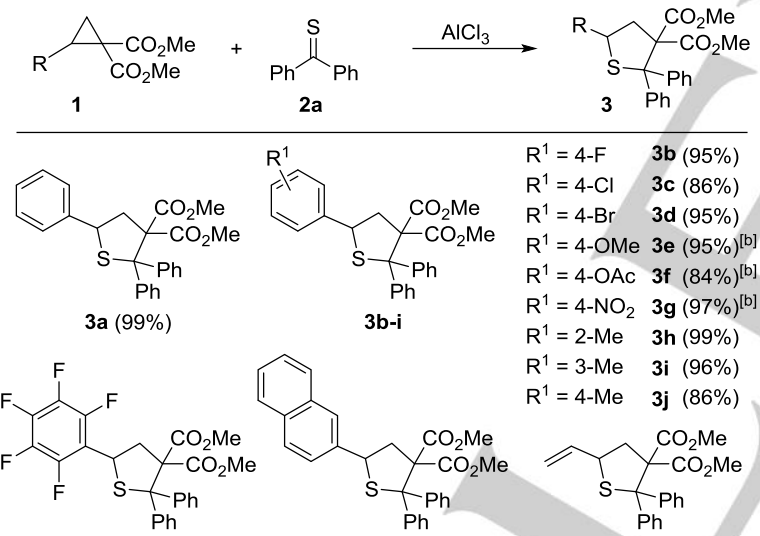

3k $(72 \%)$<smiles>COC(=O)C1(c2ccccc2)SC2CCCCC2C1(C(C)=O)c1ccccc1</smiles>

3n $(71 \%)$<smiles>COC(=O)C1(c2ccccc2)CC(N2C(=O)CCC2=O)SC1(C(C)=O)c1ccccc1</smiles>

$3 \mathbf{q}(72 \%)^{[\mathrm{b}]}$

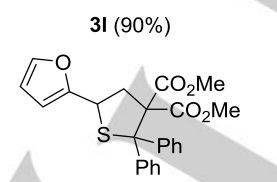

$3 m(77 \%)^{[c]}$
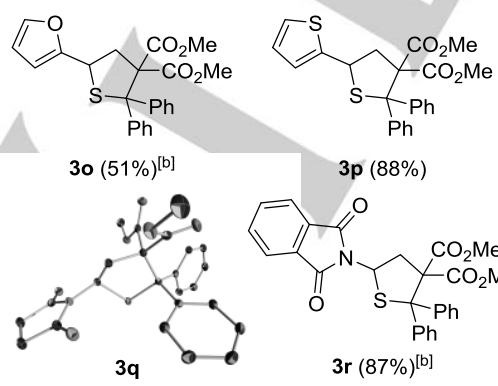

1
$3 p(88 \%)$$$
0
$$

$\mathrm{CO}_{2} \mathrm{Me}$

$\prod_{\mathrm{S}}^{\mathrm{N}} \underset{\mathrm{Ph}}{\mathrm{CO}_{\mathrm{Ph}}^{\mathrm{CO}_{2} \mathrm{Me}}}$

$3 \mathbf{r}(87 \%)^{[\mathrm{b}]}$
Scheme 2. $\mathrm{AlCl}_{3}-$ Catalyzed insertion of thiobenzophenone $2 \mathrm{a}$ into various $\mathrm{D}-\mathrm{A}$ cyclopropanes 1 leading to tetrahydrothiophenes 3 . [a] Reaction conditions: 1 $(100 \mu \mathrm{mol}), 2 \mathrm{a}(200 \mu \mathrm{mol}), \mathrm{AlCl}_{3}(10 \mathrm{~mol} \%), \mathrm{CH}_{2} \mathrm{Cl}_{2}(2 \mathrm{~mL}), 25^{\circ} \mathrm{C}, 4 \mathrm{~h}, \mathrm{Ar}$ atmosphere; yields represent isolated and purified products; [b] $\mathrm{AlCl}_{3}$ (20 mol\%); [c] $200 \mu \mathrm{mol}$ scale.

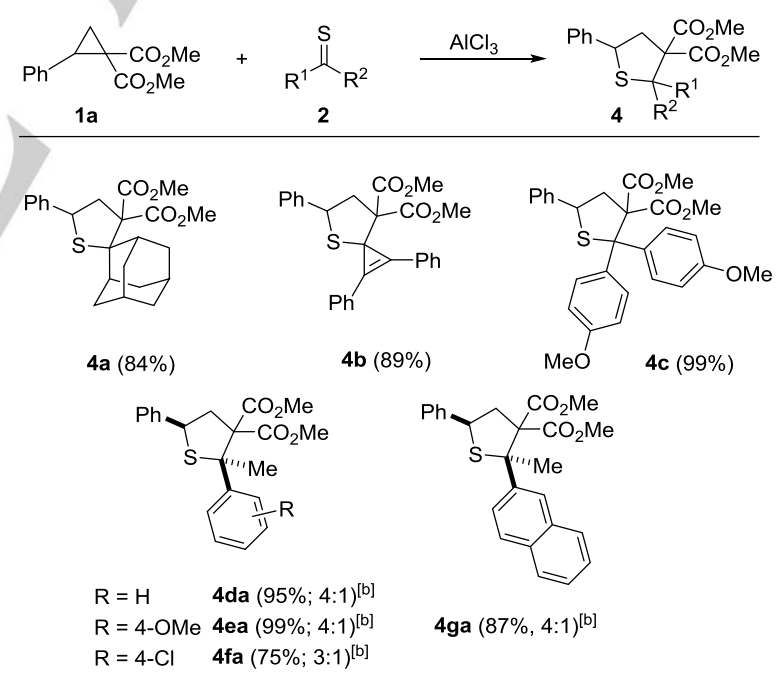

Scheme 3. $\mathrm{AlCl}_{3}$-Catalyzed insertion of various thioketones 2 in $\mathrm{D}-\mathrm{A}$ cyclopropane 1a. [a] Reaction conditions: 1a $(200 \mu \mathrm{mol}), 2(400 \mu \mathrm{mol}), \mathrm{AlCl}_{3}$ (10 mol\%), $\mathrm{CH}_{2} \mathrm{Cl}_{2}(4 \mathrm{~mL}), 25^{\circ} \mathrm{C}, 4 \mathrm{~h}, \mathrm{Ar}$ atmosphere; yields represent isolated and purified products; [b] $\mathrm{AlCl}_{3}(20 \mathrm{~mol} \%)$.

Next, we explored the stereospecificity of the [3+2]-cycloaddition by using enantioenriched cyclopropane (S)-1a (95\% ee); product 4da was formed in excellent yield with $92 \%$ ee (Scheme 4). Inversion at $\mathrm{C} 1$ was observed and confirmed by $\mathrm{X}$-ray structure elucidation. ${ }^{[18]}$ This fact strengthens the assumption that the initial 
attack of the sulfur follows an $\mathrm{S}_{\mathrm{N}}$ 2-type reaction pathway followed by ring-closure. ${ }^{[14]}$

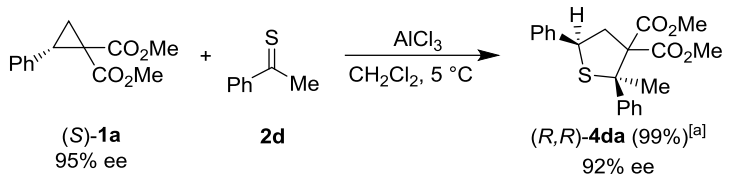

Scheme 4. Stereospecificity experiment. [a] Only the major diastereomer $(R, R)$ - $\mathbf{4 d a}$ is shown; $(S, R)-\mathbf{4 d b}$ shows similar results (see $\mathrm{SI}$ ).

These results paved the way for an intramolecular variant, resulting in bicyclic scaffolds via a simple one-pot process. Six easily available model systems $\mathbf{5}$ were generated. In all of these systems a D-A cyclopropane and a carbonyl unit (either an aldehyde or a ketone) are tethered to each other (Scheme 5). Lawesson's reagent was utilized to convert the carbonyl into a thiocarbonyl moiety. During this one-pot process bicyclic sevenand eight-membered ring systems $\mathbf{7 a - 7 f}$ comprising one sulfur bridge were formed in yields of $53-98 \%$. In the formation of sevenmembered rings, the thiocarbonyl moieties immediately inserted into the activated three-membered ring system affording oligocyclic compounds of type 7. No additional Lewis acid was required; Lawesson's reagent seems to play a dual role, as both thionation reagent and weak Lewis acid. ${ }^{[19]}$ The sulfur-bridged azepine 7c shows that even a thioaldehyde was able to undergo this reaction in moderate yield.
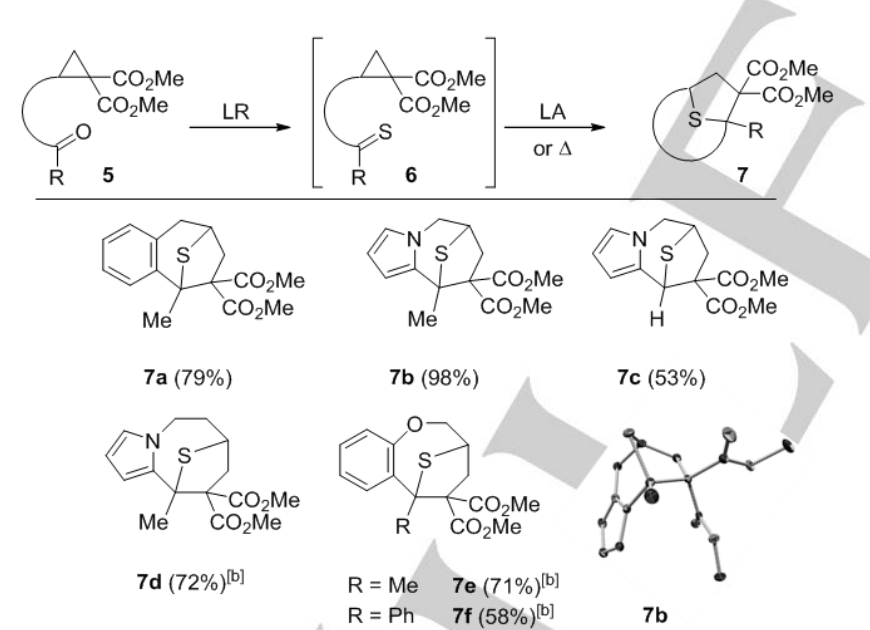

$7 b$

Scheme 5. Intramolecular reaction leading to sulfur-bridged carbo- and heterocyclic ring systems. [a] Reaction conditions: $5(100 \mu \mathrm{mol}), \operatorname{LR}(150 \mu \mathrm{mol})$, solvent $(4 \mathrm{~mL}), 90^{\circ} \mathrm{C}$, up to $12 \mathrm{~h}$; yields represent isolated and purified products. [b] For stable thioketones: $6(100 \mu \mathrm{mol}), \mathrm{AlCl}_{3}(20 \mathrm{~mol} \%), \mathrm{CH}_{2} \mathrm{Cl}_{2}(4 \mathrm{~mL}), 25^{\circ} \mathrm{C}$, $2 \mathrm{~h}$; LR = Lawesson's reagent.

Finally, our investigations were successfully extended to the synthesis of tetrahydroselenophenes by using selenoketones. ${ }^{[20]}$ Initially, we tried the same reaction conditions as used above, but with poor results. However, by changing the solvent to benzene and increasing the amount of the capricious selenoketone $\mathbf{8}$ the transformation proceeded smoothly (Scheme 6). A series of tetrahydroselenophenes $\mathbf{9 a - 9 f}$ were synthesized in moderate to good yields.

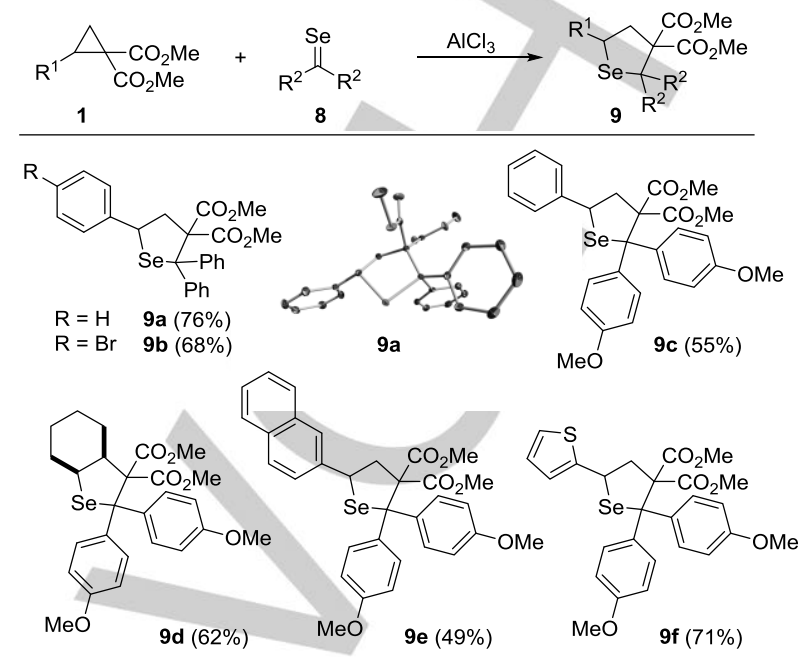

Scheme 6. $\mathrm{AlCl}_{3}-$ Catalyzed insertion of selenoketones into D-A-cyclopropanes [a] Reaction conditions: $1(100 \mu \mathrm{mol}), 8(400 \mu \mathrm{mol}), \mathrm{AlCl}_{3}(10 \mathrm{~mol} \%)$, benzene $(10 \mathrm{~mL}), 25^{\circ} \mathrm{C}$, up to $12 \mathrm{~h}$; yields represent isolated and purified products.

In summary, we have developed a novel access to tetrahydrothiophene (THT) derivatives by a Lewis-acid-catalyzed $[3+2]$-cycloaddition of thioketones and D-A cyclopropanes. A wide range of highly substituted THT derivatives was obtained in a stereospecific manner under mild conditions and high functional group tolerance. An intramolecular variant paved the way to thia[n.2.1] bicyclic ring systems. The use of selenoketones, the heavier analogs of thioketones, provided access to highly substituted tetrahydroselenophenes.

\section{Acknowledgments}

This research was supported by the European Research Council (ERC Consolidator Grant "GAINBYSTRAIN" to D.B.W.)

Keywords: cyclopropanes • donor-acceptor compounds • thiocarbonyls $\bullet$ cycloaddition $\bullet$ bicyclic systems

[1] Recent examples: a) G. Mlostoń, P. Pipiak, A. Linden, H. Heimgartner Helv. Chim. Acta 2015, 98, 462; b) G. Mlostoń, R. Hamera-Fałdyga, A. Linden, H. Heimgartner, Beilstein J. Org. Chem. 2016, 12, 1421; c) G. Mlostoń, M. K. Kowalski, E. Obijalska, H. Heimgartner, J. Fluorine Chem. 2017, 199, 92; d) G. Mlostoń, P. Grzelak, R. Hamera-Fałdyga, M. Jasiński, P. Pipiak, K. Urbaniak, Ł. Albrecht, J. Hejmanowska, H. Heimgartner, Phosphorus Sulfur Silicon Relat. Elem. 2017, 192, 204.

[2] a) G. Mlostoń, H. Heimgartner, Helv. Chim. Acta 1996, 79, 1785; b) G. Mlostoń, J. Romański, A. Swiątek, H. Heimgartner, Helv. Chim. Acta 1999, 82, 946; c) G. Mlostoń, G. K. S. Prakash, G. A. Olah, H. Heimgartner, Helv. Chim. Acta 2002, 85, 1644.

[3] a) A. Ohno, Y. Ohnishi, G. Tsuchihashi, Tetrahedron Lett. 1969, 10, 283 b) A. Ohno, Y. Ohnishi, G. Tsuchihashi, J. Am. Chem. Soc. 1969, 91, 
5038; c) H. Gotthardt, S. Nieberl, Chem. Ber. 1978, 111, 1471; d) J. W Kelly, P. L. Robinson, S. A. Evans, J. Org. Chem. 1985, 50, 5009; e) V. P. Rao, V. Ramamurthy, J. Org. Chem. 1988, 53, 332.

[4] a) J. Kamphuis, R. G. Visser, H. J. T. Bos, Tetrahedron Lett. 1982, 23, 3603; b) J. Kamphuis, A. P. Ruijter, H. J. T. Bos, J. Chem. Soc., Perkin Trans. 2 1987, 907; c) H.-B. Yang, Y.-C. Yuan, Y. Wei, M. Shi, Chem Commun. 2015, 51, 6430.

[5] a) H. Kohn, P. Charumilind, Y. Gopichand, J. Org. Chem. 1978, 43, 4961 b) G. Barbaro, A. Battaglia, P. Giorgianna, J. Org. Chem. 1987, 52, 3289 c) J. D. Coyle, P. A. Rapley, J. Kamphuis, H. J. T. Bos, J. Chem. Soc., Perkin Trans. 1 1985, 1957; d) G. Mlostoń, K. Urbaniak, A. Szychowska A. Linden, H. Heimgartner, Heterocycles 2015, 90, 529.

[6] K. Okuma, S. Sonoda, Y. Koga, K. Shioji, J. Chem. Soc., Perkin Trans. 11999, 2997.

[7] a) J. Breu, P. Höcht, U. Rohr, J. Schatz, J. Sauer, Eur. J. Org. Chem. 1998, 2861; b) U. Rohr, J. Schatz, J. Sauer, Eur. J. Org. Chem. 1998 2875; c) R. Huisgen, J. Rapp, Heterocycles 1997, 45, 507; d) J. Hejmanowska, M. Jasiński, G. Mlostoń, Ł. Albrecht, Eur. J. Org. Chem. 2017, 950

[8] M. Jasiński, G. Mlostoń, M. Stolarski, W. Costa, M. Domínguez, H.-U. Reissig, Chem. Asian J. 2014, 9, 2641.

[9] For reviews on D-A cyclopropanes: a) H.-U. Reissig, R. Zimmer, Chem. Rev. 2003, 103, 1151; b) M. Yu, B. L. Pagenkopf, Tetrahedron 2005, 61, 321 ; c) D. Agrawal, V. K. Yadav, Chem. Commun. 2008, 6471; d) C. A Carson, M. A. Kerr, Chem. Soc. Rev. 2009, 38, 3051; e) F. De Simone J. Waser, Synthesis 2009, 3353; f) M. A. Cavitt, L. H. Phun, S. France, Chem. Soc. Rev. 2014, 43, 804; g) T. F. Schneider, J. Kaschel, D. B Werz, Angew. Chem. Int. Ed. 2014, 53, 5504; Angew. Chem. 2014, 126, 5608; h) R. A. Novikov, Y. V. Tomilov, Mendeleev Commun. 2015, 25, 1

[10] M. S. Gordon, J. Am. Chem. Soc. 1980, 102, 7419.

[11] For selected examples: a) C. Brückner, H.-U. Reissig, Angew. Chem. Int. Ed. Engl. 1985, 24, 588; Angew. Chem. 1985, 97, 578; b) C. Brand, G. Rauch, M. Zanoni, B. Dittrich, D. B. Werz, J. Org. Chem. 2009, 74, 8779 c) T. F. Schneider, J. Kaschel, B. Dittrich, D. B. Werz, Org. Lett. 2009, 11, 2317; d) S. J. Gharpure, M. K. Shukla, U. Vijayasree, Org. Lett. 2009 11, 5466; e) T. F. Schneider, J. Kaschel, S. I. Awan, B. Dittrich, D. B. Werz, Chem. Eur. J. 2010, 16, 11276; f) J. Kaschel, T. F. Schneider, D. Kratzert, D. Stalke, D. B. Werz, Angew. Chem. Int. Ed. 2012, 51, 11153; Angew. Chem. 2012, 124, 11315; g) H. K. Grover, M. R. Emmett, M. A. Kerr, Org. Lett. 2013, 15, 4838; h) P. Wang, S. Song, Z. Miao, G. Yang, A. Zhang, Org. Lett. 2013, 15, 3852; i) C. D. Schmidt, J. Kaschel, T. F. Schneider, D. Kratzert, D. Stalke, D. B. Werz, Org. Lett. 2013, 15, 6098.

[12] For selected examples: a) H.-U. Reissig, E. Hirsch, Angew. Chem. Int. Ed. Engl. 1980, 19, 813, Angew. Chem. 1980, 92, 839; b) L. K. B. Garve, P. Barkawitz, P. G. Jones, D. B. Werz, Org. Lett. 2014, 16, 5804; c) K. L. Ivanov, E. V. Villemson, E. M. Budynina, O. A. Ivanova, I. V. Trushkov, M. Y. Melnikov, Chem. Eur. J. 2015, 21, 4975; d) Y. Xia, X. Liu, H. Zheng, L. Lin, X. Feng, Angew. Chem. Int. Ed. 2015, 54, 227; Angew. Chem. 2015, 127, 229; e) Y. Xia, L. Lin, F. Chang, Y. Liao, X. Liu, X. Feng, Angew. Chem. Int. Ed. 2016, 55, 12228; Angew. Chem. 2016, 128 12416; f) S. Das, C. G. Daniliuc, A. Studer, Org. Lett. 2016, 18, 5576; g) T. Kaicharla, T. Roy, M. Thangaraj, R. G. Gonnade, A. T. Biju, Angew. Chem. Int. Ed. 2016, 55, 10061; Angew. Chem. 2016, 128, 10215; h) C Ross Pitts, B. Ling, J. A. Snyder, A. E. Bragg, T. Lectka, J. Am. Chem. Soc. 2016, 138, 6598; i) J. Wallbaum, L. K. B. Garve, P. G. Jones, D. B. Werz, Org. Lett. 2017, 19, 98; j) L. K. B. Garve, P. G. Jones, D. B. Werz, Angew. Chem. Int. Ed. 2017, 56, 9226; Angew. Chem. 2017, 129, 9354; k) S. Das, C. G. Daniliuc, A. Studer, Angew. Chem. Int. Ed. 2017, 56
ASAP; Angew. Chem. 2017, 129, ASAP; I) A. Lücht, L. J. Patalag, A. U Augustin, P. G. Jones, D. B. Werz, Angew. Chem. Int. Ed. 2017, 56, 10587; Angew. Chem. 2017, 129, 10723.

[13] For selected examples: a) S. Xing, W. Pan, C. Liu, J. Ren, Z. Wang, Angew. Chem. Int. Ed. 2010, 49, 3215; Angew. Chem. 2010, 122, 3283; b) S. Xing, Y. Li, Z. Li, C. Liu, J. Ren, Z. Wang, Angew. Chem. Int. Ed. 2011, 50, 12605; Angew. Chem. 2011, 123, 12813; c) J.-P. Qu, Y. Liang, H. Xu, X.-L. Sun, Z.-X. Yu, Y. Tang, Chem. Eur. J. 2012, 18, 2196; d) H Xu, J.-P. Qu, S. Liao, H. Xiong, Y. Tang, Angew. Chem. Int. Ed. 2013, 52, 4004; Angew. Chem. 2013, 125, 4096; e) A. F. G. Goldberg, N. R. O' Connor, R. A. Craig II, B. M. Stoltz, Org. Lett. 2012, 14, 5314; f) W. Zhu, J. Fang, Y. Liu, J. Ren, Z. Wang, Angew. Chem. Int. Ed. 2013, 52, 2032; Angew. Chem. 2013, 125, 2086; g) S. Chakrabarty, I. Chatterjee, B. Wibbeling, C. G. Daniliuc, A. Studer, Angew. Chem. Int. Ed. 2014, 53, 5964; Angew. Chem. 2014, 126, 6074; h) D. Gladow, H.-U. Reissig, J. Org. Chem. 2014, 79, 4492; i) J. Ren, J. Bao, W. Ma, Z. Wang, Synlett 2014, 25, 2260; j) H. Xu, J.-L. Hu, L. Wang, S. Liao, Y. Tang, J. Am. Chem. Soc. 2015, 137, 8006; k) S.-W. Wang, W.-S. Guo, L.-R. Wen, M. Li, RSC Adv. 2015, 5, 47418; I) L. K. B. Garve, M. Petzold, P. G. Jones, D. B. Werz, Org. Lett. 2016, 18, 564; m) A. Gosh, S. Mandal, P. K. Chattaraj, P. Banerjee, Org. Lett. 2016, 18, 4940; n) L. K. B. Garve, M. Pawliczek, J. Wallbaum, P. G. Jones, D. B. Werz, Chem. Eur. J. 2016, 22, 521; o) J. Sabbatani, N. Maulide, Angew. Chem. Int. Ed. 2016, 55, 6780; Angew. Chem. 2016, 128, 6892; p) D. D. Borisov, R. A. Novikov Y. V. Tomilov, Angew. Chem. Int. Ed. 2016, 55, 12233; Angew. Chem 2016, 128, 12421; q) Z.-H. Wang, H.-H. Zhang, D.-M. Wang, P.-F. Xu, Y.-C. Luo, Chem. Commun. 2017, 53, 8521

[14] a) P. D. Pohlhaus, J. S. Johnson, J. Am. Chem. Soc. 2005, 127, 16014 b) P. D. Pohlhaus, S. D. Sanders, A. T. Parsons, W. Li, J. S. Johnson, J Am. Chem. Soc. 2008, 130, 8642; c) A. T. Parsons, J. S. Johnson, J. Am. Chem. Soc. 2009, 131, 3122

[15] a) F. Benfatti, F. de Nanteuil, J. Waser, Org. Lett. 2012, 14, 386; b) F. Benfatti, F. de Nanteuil, J. Waser, Chem. Eur. J. 2012, 18, 4844; c) F. de Nanteuil, E. Serrano, D. Perrotta, J. Waser, J. Am. Chem. Soc. 2014, 136, 6239; d) S. Racine, F. de Nanteuil, E. Serrano, J. Waser, Angew. Chem. Int. Ed. 2014, 53, 8484; Angew. Chem. 2014, 126, 8624.

[16] F.de Nanteuil, J. Waser, Angew. Chem. Int. Ed. 2011, 50, 12075; Angew. Chem. 2011, 123, 12281.

[17] S. Racine, B. Hegedüs, R. Scopelliti, J. Waser, Chem. Eur. J. 2016, 22, 11997.

[18] The inversion of the configuration was proved by the preparation of $(R)$-3a, which was synthesized under the same conditions, but did not show a complete baseline separation during chiral HPLC for exact determination of the ee.

[19] J. Kaschel, C. D. Schmidt, M. Mumby, D. Kratzert, D. Stalke, D. B. Werz, Chem. Commun. 2013, 49, 4403.

[20] G. Erker, R. Hock, C. Krüger, S. Werner, F.-G. Klärner, U. ArtschagerPerl, Angew. Chem. Int. Ed. Engl. 1990, 29, 1067; Angew. Chem. 1990 , 102, 1082.

[21] a) CrysAlisPro; Agilent Technologies: Oxford, U.K. 2013; b) G. M. Sheldrick, SHELXL-97, University of Göttingen, Germany, 1997; c) G. M. Sheldrick, Acta Crystallogr. Sect. A 2008, 64, 112

[22] The CIF files have been deposited with the Cambridge Crystallographic Data Centre as supplementary publication no. CCDC-1568335 ((R)-3a) -1568336 (3h), -1568337 (3i), -1568338 (3q), -1568339 (7b), -1568340 (7f) and $-1568341(9 a)$. These data can be obtained free of charge from The Cambridge Crystallographic Data Centre. 


\section{COMMUNICATION}

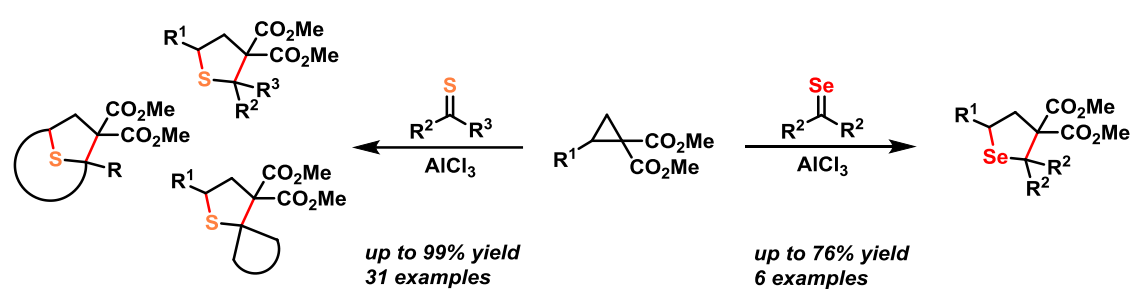

Thiocarbonyl Insertion: Donor-Acceptor cyclopropanes undergo a formal [3+2]cycloaddition with thioketones using $\mathrm{AlCl}_{3}$ catalysis under mild conditions to yield highly substituted tetrahydrothiophenes. Extension to an intramolecular variant led to bicyclic products. Similar cycloadditions with bisarylselenoketones were also achieved.
A. U. Augustin, M. Sensse, P. G. Jones, D. B. Werz*

Page No. - Page No.

Stereospecific Reaction of DonorAcceptor Cyclopropanes with Thioketones: A Novel Access to Highly Substituted Tetrahydrothiophenes 